\title{
Acute mania after levothyroxine replacement for hypothyroid-induced heart block
}

\author{
Marc Gregory Yu, ${ }^{1}$ Karen Marie Flores, ${ }^{2}$ Iris Thiele Isip-Tan ${ }^{2}$
}

${ }^{1}$ Department of Medicine, Section of Endocrinology, Diabetes and Metabolism, Manila, Philippines

${ }^{2}$ Department of Medicine, University of the PhilippinesPhilippine General Hospital, Manila, Philippines

\section{Correspondence to}

Dr Marc Gregory Yu, marcgreggy@yahoo.com

Accepted 4 January 2017
CrossMark

To cite: Yu MG, Flores KM,
Isip-Tan IT. BMJ Case Rep
Published online: [please
include Day Month Year]
doi:10.1136/bcr-2016-
218819

To cite: Yu MG, Flores KM, Isip-Tan IT. BMJ Case Rep include Day Month Year 218819

\section{SUMMARY}

Psychiatric disturbances can manifest after levothyroxine (LT4) treatment for severe hypothyroidism. We present the case of a young Filipino man with Hashimoto's thyroiditis and high-grade heart block, who was given a full replacement LT4 dose on admission. Twenty-four hours after this dose, he developed manic symptoms, which were addressed with sedatives and neuroleptics with gradual restoration of euthymia the following day. A comprehensive workup did not reveal any findings suggestive of another aetiology for either mania or heart block. We ultimately ascribed the mania as secondary to LT4, and the heart block to hypothyroidism. Although mania is more likely to be precipitated by high starting LT4 doses, reports have shown that symptoms can still arise even at lower doses and with more gradual titration, especially in long-standing hypothyroidism.

\section{BACKGROUND}

While psychiatric signs and symptoms are welldocumented manifestations of hypothyroidism, rapid normalisation to a euthyroid state can also present in a similar manner. This report aims to emphasise the importance of recognising and managing acute mania after LT4 therapy for a hypothyroid patient with cardiac complications, as well as the need for an extensive workup to rule out other aetiologies.

\section{CASE PRESENTATION}

A 34-year-old Filipino man presented at the emergency department with a long-standing anterior neck mass associated with cold intolerance, constipation, insomnia, weight gain and dry, puffy skin. He was previously diagnosed with primary hypothyroidism and prescribed LT4 but failed to take his medications and was lost to follow-up. The patient had no comorbidities, had an unremarkable family history and had no prior intake of any drug known to interfere with thyroid function. On admission, he was normothermic, normotensive, bradycardic with a pulse rate of $50 \mathrm{bpm}$ and hyporeflexive. An ECG showed high-grade atrioventricular (AV) block. Thyroid function tests revealed low free thyroxine at $5.15 \mathrm{pmol} / \mathrm{L}$ (normal 9.01$19.05 \mathrm{pmol} / \mathrm{L})$, low free triiodothyronine at $<1.54 \mathrm{pmol} / \mathrm{L}$ (normal $2.63-5.67 \mathrm{pmol} / \mathrm{L}$ ) and elevated thyroid-stimulating hormone (TSH) at $>100 \mu \mathrm{IU} / \mathrm{mL}$ (normal $0.35-494 \mu \mathrm{IU} / \mathrm{mL}$ ). LT4 was started at the full-replacement dose of $100 \mathrm{mcg}$ $(1.6 \mathrm{mcg} / \mathrm{kg})$, and the patient was referred to cardiology. However, 24 hours after the first LT4 dose, he developed agitation, irritability and restlessness, with increased talking speed and volume and flight of ideas. He was easily distracted, refused to sleep during the night and attempted to leave the hospital. A repeat ECG showed no change from baseline. Subsequently, a stat referral to psychiatry was made.

\section{INVESTIGATIONS}

The diagnosis of Hashimoto's thyroiditis was established with the presence of elevated thyroid peroxidase antibodies (anti-TPO) at $3771 \mathrm{U} / \mathrm{mL}$ (normal $<100 \mathrm{U} / \mathrm{mL}$ ) and elevated thyroglobulin antibodies (anti-Tg) at $9449 \mathrm{IU} / \mathrm{mL}$ (normal $<50 \mathrm{IU} / \mathrm{mL}$ ). Neck ultrasound scan showed an enlarged thyroid with heterogeneous parenchymal echopattern and multiple subcentimeter hypoechoic foci, also consistent with Hashimoto's thyroiditis. Psychiatric evaluation fulfilled the DSM-IV criteria for manic episode (table 1). The aetiology of the mania was thoroughly investigated by performing serum electrolytes, a complete blood count, a toxicology panel, an EEG and cranial MRI, which all turned out normal. A cerebrospinal fluid (CSF) examination to rule out central nervous system (CNS) infection was also performed revealing unremarkable results. For the patient's heart block, additional workup consisted of cardiac creatine kinase (CK-MB) and troponin I, both with normal levels. Two-dimensional (2D) echocardiogram with Doppler, on the other hand, showed concentric left ventricular hypertrophy with good wall motion and contractility and preserved overall systolic function (ejection fraction by Teicholz 56\%, Simpson's $55 \%$ ) with Doppler evidence of diastolic dysfunction.

\section{TREATMENT}

Haloperidol was administered for the mania followed by a low-dose midazolam drip, with the patient carefully monitored for QT prolongation and hypotension. The following day, he was found awake, calm, cooperative, coherent and oriented to three spheres, with no evidence of irritability or agitation. Haloperidol and midazolam were both gradually withdrawn, and LT4 was continued at the current dose. For the heart block, the cardiology service opted for close observation, with standby atropine and pacemaker insertion should his condition deteriorate.

\section{OUTCOME AND FOLLOW-UP}

The patient remained stable throughout the rest of his admission, with no relapse of psychiatric symptoms or progression of heart block, and was 
Table 1 DSM-IV criteria for a manic episode and the criteria fulfilled by the patient

\begin{tabular}{l}
\hline DSM-IV criteria for manic episode \\
\hline $\begin{array}{l}\text { 1. A distinct period of abnormally and persistently elevated or irritable } \\
\text { mood, lasting at least } 1 \text { week, or any duration if hospitalised } \\
\text { fulfilled }\end{array}$ \\
2. During the period of mood disturbance, three (or more) of the \\
following have persisted (four if the mood is only irritable) and \\
have been present to a significant degree: \\
Inflated self-esteem or grandiosity \\
$\quad$ Decreased need for sleep \\
More talkative than usual \\
$\quad$ Flight of ideas or subjective experience that thoughts are racing \\
$\quad$ Distractibility
\end{tabular}

eventually discharged on the ninth hospital day. He was advised to follow-up at the outpatient department for thyroid function monitoring, cardiac evaluation and psychiatric assessment.

\section{DISCUSSION}

Psychotic disorders (so-called 'thyroid psychoses') have long been described in patients with either hypothyroidism or hyperthyroidism. Although hypothyroidism has been anecdotally associated with depression and hyperthyroidism with mania, acute mania can occur in hypothyroidism. ${ }^{1}$ Likewise, mania can also be precipitated by thyroid hormone intake, as described in some hypothyroid patients who developed the condition shortly after initiating LT4 replacement. ${ }^{2-4}$

Our patient, a young hypothyroid man with no prior psychiatric illness, presented with manic symptoms 24 hours after starting LT4. Owing to the temporal relationship between LT4 intake and onset of psychiatric manifestations, as well as the lack of significant findings on history and workup, our primary impression for the mania was LT4-induced. Our other differential was Hashimoto's encephalopathy, given the high titres of anti-TPO and anti-Tg, supportive neck ultrasound scan findings, unremarkable CSF findings (which are found in 25\% of cases) and normal cranial MRI (which may also show cerebral atrophy, dural enhancement or non-specific T2-weighted signal abnormalities in the subcortical white matter). ${ }^{56}$

However, Hashimoto's encephalopathy tends to affect older patients, is more common in women, presents subacutely rather than acutely and manifests predominantly with stroke-like episodes, seizures, epilepsy and myoclonus, as opposed to mania. Another important consideration is the fact that in our patient, the psychiatric symptoms appeared after, and not before, LT4 administration. Moreover, Hashimoto's encephalopathy requires treatment with high-dose steroids (hence the term 'steroid-responsive encephalopathy'); our patient's improvement without the need for steroids strongly argues against this diagnosis. ${ }^{7}$ Finally, rapid complete resolution of psychiatric symptoms-within 24 hours in this case-is uncharacteristic of
Hashimoto's encephalopathy, which tends to have a more prolonged course lasting several weeks regardless of treatment. ${ }^{2}$

The pathophysiology of LT4-induced mania is thought to be due to the rapid alteration of thyroid function rather than to LT4 itself, especially since instances of T3-induced mania have also been observed in patients with drug-resistant bipolar depression. ${ }^{8}$ In addition, antithyroid drugs have been occasionally reported to cause similar phenomena. ${ }^{9}$ It is hypothesised that sudden fluctuations in thyroid status lead to variations in catecholamine metabolism and abruptly augment postsynaptic catecholamine receptor sensitivity, thereby precipitating a hyperadrenergic state and increasing the risk of manic and psychotic symptoms. The same is true for cases of iatrogenic hypothyroidism. ${ }^{10}$

In the various case reports on LT4-induced mania, most patients were women and had either a personal or family history of psychosis. ${ }^{2}$ The onset of manic symptoms ranged from as short as 24 hours (as in our patient) to as long as 3 weeks after the first LT4 dose, with cases resolving after 24 hours (as in our patient) to several months, either on their own or after temporary cessation of LT4 therapy from 5 days to 2 weeks. $^{2-4}$ These reports also noted that while the average initial dose of LT4 was relatively high $(180 \mathrm{mcg} / \mathrm{d})$, the psychiatric disturbances can also manifest at lower doses $(25-50 \mathrm{mcg})$ and even with gradual dose titration. ${ }^{3}{ }^{4}$ Hence, in retrospect, a lower starting LT4 dose may have lessened-but not completely avoided-the probability of precipitating manic symptoms.

For our patient, the reasons for the full replacement LT4 dose were his young age, profound hypothyroidism and absence of comorbidities. The presence of high-grade heart block was eventually attributed to hypothyroidism after an unremarkable cardiac workup and supportive 2D echocardiogram features. ${ }^{11}$ Hypothyroidism remains one of the rare AV block aetiologies where complete recovery under pharmacological management is possible, although full recovery of the conduction system usually takes several days to weeks. ${ }^{12}$ It is precisely because of this full reversibility that the cardiology team opted for close observation rather than outright pacemaker insertion.

As of writing, the patient is yet to follow-up at the outpatient department with the results of his latest thyroid function tests. Despite normalisation of serum thyroid hormone levels, normalisation of TSH may variably lag after severe hypothyroidism or hyperthyroidism. This phenomenon is called hysteresis of the hypothalamus-pituitary-thyroid axis. ${ }^{13}$

Our case is significant in that it is the first report involving a double-edged sword: dealing with manic symptoms from LT4 administration at the same needing to correct severe

\section{Learning points}

- Hypothyroidism and its treatment with LT4 can have psychiatric manifestations.

- In hypothyroid patients with psychiatric and cardiac manifestations, an exhaustive workup is needed to rule out other diagnoses.

- Conversely, in patients presenting with unexplained psychiatric disturbances and heart block, thyroid function testing is also essential.

- The pros and cons of treatment should be carefully considered, and timely referrals should be made. 
hypothyroidism complicated by heart block. This illustrates the importance of a comprehensive workup to rule out other aetiologies for both conditions, sound clinical judgement to weigh the risks and benefits of treatment, and timely referrals to appropriate subspecialty services. Close monitoring of the patient's day-to-day status is emphasised.

Contributors MGY and KMF were responsible for writing the manuscript draft. ITI-T was responsible for editing the manuscript draft and approving the final version. All authors were involved in the management of the patient.

Competing interests None declared.

Patient consent Obtained.

Provenance and peer review Not commissioned; externally peer reviewed.

\section{REFERENCES}

1 Khemka D, Ali JA, Koch CA. Primary hypothyroidism associated with acute mania: L case series and literature review. Exp Clin Endocrinol Diabetes 2011;119:513-17.

2 Josephson AM, Mackenzie TB. Thyroid-induced mania in hypothyroid patients. $\mathrm{Br}$ J Psychiatry 1980;137:222-8.
3 El-Kaissi S, Kotowicz MA, Berk M, et al. Acute delirium in the setting of primary hypothyroidism: the role of thyroid hormone replacement therapy. Thyroid 2005; 15:1099-101.

4 Verma R, Sachdeva A, Singh $Y$, et al. Acute mania after thyroxin supplementation in hypothyroid state. Ind J Endocrinol Metab 2013;17:922-3.

5 Song YM, Seo DW, Chang GY. MR findings in Hashimoto's encephalopathy. Am J Neuroradiol 2004;25:807-8.

6 Takahashi S, Mitamura R, Itoh Y, et al. Hashimoto encephalopathy: etiologic considerations. Pediatr Neurol 1994;11:328-31.

7 Mocellin R, Walterfang M, Velakoulis D. Hashimoto's encephalopathy: epidemiology, pathogenesis and management. CNS Drugs 2007;21:799-811.

8 Evans DL, Strawn SK, Haggerty JJ. Appearance of mania in drug-resistant bipolar depressed patients after treatment with L-triiodothyronine. Clin Psychiatry 1986;47:521-2.

9 Vita R, Mazzi V, Antonelli A, et al. Antithyroid drugs and psychosis. Expert Opin Drug Saf 2013;12:865-72.

10 Benvenga S, Lapa D, Trimarchi F. Don't forget the thyroid in the etiology of psychoses. Am J Med 2003;115:159-60.

11 Rawat B, Satyal A. An echocardiographic study of cardiac changes in hypothyroidism and the response to treatment. Kathmandu Univ Med J 2003;2:182-7.

12 Seol SH, Kim DI, Park BM, et al. Complete atrioventricular block presenting with syncope caused by severe hypothyroidism. Cardiol Res 2012;3:239-41.

13 Leow MKS. A review of the phenomenon of hysteresis in the hypothalamus-pituitary-thyroid axis. Front Endocrinol (Lausanne) 2016;7:64.

Copyright 2017 BMJ Publishing Group. All rights reserved. For permission to reuse any of this content visit

http://group.bmj.com/group/rights-licensing/permissions.

BMJ Case Report Fellows may re-use this article for personal use and teaching without any further permission.

Become a Fellow of BMJ Case Reports today and you can:

- Submit as many cases as you like

- Enjoy fast sympathetic peer review and rapid publication of accepted articles

- Access all the published articles

- Re-use any of the published material for personal use and teaching without further permission

For information on Institutional Fellowships contact consortiasales@bmjgroup.com

Visit casereports.bmj.com for more articles like this and to become a Fellow 